\title{
The Secondary Effects of Secondary Gains: A Case Report of Conversion Disorder
}

\author{
Srinidhi CV*, A G Shanthi and Smitha Ruckmani \\ Department of Clinical Psychology, Institute of Mental Health, Chennai, India
}

Submission: October 22, 2019; Published: November 12, 2019

*Corresponding author: Srinidhi CV, Department of Clinical Psychology, Institute of Mental Health, Chennai, India

\begin{abstract}
A partial or complete loss of the normal integration between memories of the past, awareness of identity, immediate sensations, and control of bodily movements is termed as dissociative disorders, according to ICD-10. Initially called Hysteria, it was later renamed as conversion disorder by Sigmund Freud, owing to the conversion of psychological distress into physical symptoms. This disorder has a higher prevalence in women and in lower socio-economic status. In this case report, a psychoanalytic and behavioral model is used to explain the dissociative convulsions. Other than the secondary gains that usually accompany this disorder, the catharsis of anxiety has increased chances of pregnancy.
\end{abstract}

Keywords: Conversion Disorder; Somatization; Pseudo-seizures; Pregnancy; Dissociative convulsions

\section{Introduction}

Conversion disorder, according to ICD 10, is a dissociative disorder that involves partial or complete loss of the normal integration between memories of the past, awareness of identity, immediate sensations, and control of bodily movements. The diagnostic criterion includes no evidence of physical or bodily impairment along with evidence of a possible psychological stressor. Dissociative Convulsions, or pseudo seizures may present a clinical picture of movements, but tongue-biting, serious bruising due to falling, and incontinence of urine are rare in dissociative convulsion, and loss of consciousness is absent or replaced by a state of stupor or trance (WHO, 1984). Hippocrates was the first person to use the term 'Hysteria' to describe these cluster of symptoms. He believed that it was caused because of the movement of the uterus in the body, or the "wandering uterus" [1]. The term conversion disorder was coined by Freud. He posits that conversion disorder occurs due to somatization of the anxiety that is present in the subconscious because of the conflicts within self $[2,3]$. Conversion disorder was found to be higher in women and in young adulthood from lower socioeconomic statuses [4]. In a general hospital, $20-25 \%$ of patients showed conversion symptoms and 5\% met with the full criteria [5]. Understandably this percentage increases in neurology departments. Further, the prevalence is higher in women than men and up to $31 \%$ in prevalent in third world countries [6].

The clinical picture of conversion may vary across cultures. The most identifying features are both primary and secondary gains in people with conversion disorder. The precipitating factor is commonly easy to identify and has a temporal correlation with the onset of the symptoms. The primary gain is explained through Psychodynamic theory. According to Freud, a person's Id, or the pleasure principle and Super ego or the morality principle, are in a state of constant conflict with each other. A stressor will exacerbate this conflict and resulting in increased psychic anxiety. The ego, or the reality principle, attempts to resolve this conflict based on the societal norms and situations. Immature defenses suggest poor ego strength. Moreover, unsuccessful resolution could result in anxiety that is somatized. In this way there is a cathartic effect of conversion on the anxiety. The secondary gain is more subtle in nature $[2,6]$. The learning theory explains the secondary gain through reinforcement. The person, due to their sickness is not only relieved from usual responsibilities and decision making with respect to the stressful situation, but also receives increased care and attention from their loved ones. This becomes a reinforcer or a reward they receive for having these symptoms. As a result of this, the probability of the symptoms recurring increases [5,7].

\section{Case Summary}

Mrs. M was a 28-year-old married female from a low socioeconomic status with complaints of episodes of fainting for the past one and a half years and seizure-like attack for the past one year. $\mathrm{M}$ was apparently normal till 1 and a half years back when she had an episode of fainting when she and her husband were riding in a bike to her hometown. She was going to visit her father in the afternoon and was the pillion. She leaned on her husband and said she felt dizzy. Her husband parked the bike alongside of the road. She had fainted by 
then. Around 30 seconds to one minute later, she regained consciousness after her husband splashed her face with water. She reported that she was stressed over the past few weeks before the episode. She was facing a lot of criticism that she was not able to conceive even after 1 and a half years of marriage. Though she was undergoing treatment for the cysts in her uterus, no one in her husband's family was supportive of her. They complained about her inability to conceive a baby and called names to her on her face.

The second episode happened within the same month when she fainted 2-3 times in the same day. Each time she would fall or collapse for 4-5 seconds and then again consciousness with normal levels of functioning. She was able to hear the people around her calling out her name. There were no reported instances of bruising or harming of self out of fainting. She was consequently admitted in GH for 3 days. Her medical reports indicated normal levels of brain activity; no abnormality was detected. She was prescribed Tablet Diazepam which she took for 2 weeks and then stopped as the episodes continued to occur during the period of medication. 6 months after the first episode, she began to have tonic-clonic seizure episodes as well. These episodes occurred when she is alone or around people. Since then M has had 3-4 episodes of seizure every fortnight. Urinary incontinence, frothing or tongue-biting were not present during the seizures. There was no report of pre-ictal symptoms like aura, déjà vu, hallucinations etc. Post-ictal symptoms like drowsiness, migraine, confusion and disorientation were also absent. She reported increased levels of care from her husband since episodes started. Furthermore, M conceived 7 months after onset of episodes and reported decline in frequency of episodes. $M$ is currently 8 months pregnant. After pregnancy, the in-laws continue to occasionally pass comment in front of the $\mathrm{M}$ that she is having a child very late. She was referred from Institute of Obstetrics and Gynaecology for diagnostic clarification owing to negative finding in EEG, CT, MRI of the brain.

M was first born, delivered through normal vaginal delivery, full term, normal birth weight. M's mother committed suicide 3 months after her birth and M grew up with her maternal grandparents, away from her father who was a farmer. There were no significant physical illnesses or psychiatric illness. She got married in 2014 when she was 23. She has a cordial relationship with her husband. They were trying to get pregnant since 2015 however they could not as there was a cyst in her uterus. After some treatment, M conceived in July 2018. However, during the period in between $M$ faced a lot of stress due to the delay in pregnancy. The mother-in-law, sisters and brother-in-law were critical about the scenario and would pass comments in front of the $\mathrm{M}$ regarding the same. After pregnancy, they continue to occasionally pass comment in front of the $\mathrm{M}$ that she is having a child very late. $\mathrm{M}$ was submissive and extremely adjustable in a relationship.
She doesn't have many friends. She is shy, timid, sensitive but emotionally controlled. She is quite and restrained.

Psychological test findings showed that her IQ of 91 indicates average level of performance intelligence. She got scores indicative of moderate level of depression in Hamilton Rating Scale for Depression. Significant conflicts in the area of Self-concept with respect to lack of confidence and feelings of worry, guilt related to her late conception, and conflicts within laws were elicited in the Sentence completion test. She also resents not being able to spend time with her father. In the Thematic Apperception Test, her stories indicated that the hero usually had feelings of self-doubt, helplessness, anticipatory anxiety, uncertainty, insecurity. He was usually submissive and indecisive in situations and showed elements of loneliness. Needs of nurturance, secureness, Affiliation was predominantly expressed and presses of poverty, rejection, danger, aggression was seen. The environment was conceived to be hostile, overpowering, uncertain and dangerous in the most stories. The stories had largely a negative or uncertain ending indicating $M$ has a pessimistic view of the world. Defenses that were employed principally were rationalization or acceptance. This is suggestive of poor ego integration and strength. Though $M$ has needs that are unmet, the conception of environment as overpowering and hero being help-less has led to a situation where the needs are not expressed in majority of the stories. In Rorschach Ink Blot Test, her responses suggested that $\mathrm{M}$ has high need to differentiate perceptual experiences and has difficulty in integration of reality. She prefers sticking to facts out of fears of insecurity. A constrictive manner of interpretation with little or no acknowledgement and expression of emotional needs was seen. Content analysis shows disturbed adjustment, stereotyped and narrow-minded view of the world as well as marked levels of psychic anxiety as evidenced by high levels of animal, nature and anatomical responses. The presence of a seizure-like attack and rejection of a response- both in card 2 indicates strong emotional reaction to female genital organs; emphasis on midline responses suggests she strives for safety and security. M meets the Diagnostic criteria for F.44.5 Dissociative Convulsions according to ICD-10.

\section{Case Formulation}

The case will be formulated using the psychodynamic and behavioral model. The patient's mother died, 3 months after her birth and she grew up with her grandparents. M did not have a consistent primary caregiver as couldn't spend adequate time with her father. This inconsistence in a caregiver is usually attached with insecure attachment style, features of which can be seen in the Rorschach protocol. Insecure attachment is usually associated with low self-esteem as can be seen in M. It could also have been due her grandparents who were authoritarian in parenting style. The warmth, support and positive regard was not obtained, resulting in poor self- 
concept. Furthermore, due to the poor economic condition of the family, most of M's needs were left unmet. Her id was underdeveloped owing to this. Her super ego will be strong from the values and morals that her grandparents imparted to her through their strict norms and severe punishments when mistakes were made. Ego of the patient did not fully develop due to her strong super ego and poor self-concept, along with her insecure feelings. She would often feel inadequate to make decisions as a result of the poor self-esteem. Her poor ego strength would lead to use of immature defense mechanisms when intrapsychic conflicts arises as seen from her poor story formation in TAT and employment of defenses for conflict resolution in stories.

As an adult, after her marriage the delay in her pregnancy exaggerated her feeling of low self-esteem and insecure feelings. Living in a joint family with several relatives, $M$ did not have opportunity to spend quality time with her husband, which could have increased her feeling of insecurity and did not provide her necessary comfort. She thus continued to have her anxiety levels increase. Her underdeveloped Id could not express the need for his affection successfully as well. These feelings were supressed by her super ego. At present, the patient faces criticism from her in-laws directed towards her physical self and abilities. Her low self-concept, and poor Id development will prevent her from being assertive and expressing her feelings. Instead, her super ego might dwell on her in-law's comments to induce increased levels of selfdoubt. As a result of this, the anxiety builds up without being resolved. The super ego repeatedly overpowered Id at the cost of unresolved needs. These need induced anxiety eventually surfaced to the consciousness in the form of somatization; in the patient's case, as a seizure-like attack or fainting episode. The anxiety has been channelized in this method. This is the primary gain obtained by the patient. After having released the anxiety, the seizure-like attack had resulted in increased care towards her. This increased expression of love, warmth and care she experiences fulfils her need of nurturance and affiliation without requiring her to express the need directly. This fulfilment serves a secondary reinforcement to her behaviour, increasing the likelihood of another seizure-like attack. She became pregnant after the onset of these symptoms which led to increased care from her husband. There is a reported decrease in the frequency and intensity of seizures to support this formulation as well. In this manner she continues to somatise her anxiety without proper conflict resolution, and her secondary gain facilitated a secondary effect of pregnancy.

The ideal course of management would be to psycho-educate her immediate family to reduce the secondary reinforcement or increased care she is receiving due to her seizure-like attack. The patient needs to be given psychotherapy focusing on her assertiveness skills and coping techniques as a short-term goal and resolution of intrapsychic conflicts on a long-term goal.

\section{References}

1. Tasca C, Rapetti M, Carta MG, Fadda B (2012) Women and hysteria in the history of mental health. Clinical Practice and Epidemiology in Mental Health: CP \& EMH 8: 110-119.

2. Ford CV, Folks DG (1985) Conversion disorders: An overview. Psychosomatics 26(5): 371-374.

3. Nowak DA, Fink GR (2009) Psychogenic movement disorders: Aetiology, phenomenology, neuroanatomical correlates and therapeutic approaches. NeuroImage 47(3): 1015-1025.

4. Folks DG, Ford CV, Regan WM (1984) Conversion symptoms in a general hospital. Psychosomatics 25(4): 285-295.

5. Feinstein A (2011) Conversion disorder: Advances in our understanding. Cmaj 183(8): 915-920.

6. Ali S, Jabeen S, Pate RJ, Shahid M, Chinala S, et al. (2015) Conversion Disorder-Mind versus Body: A Review. Innovations in Clinical Neuroscience 12(5-6): 27-33.

7. Owens C, Dein S (2006) Conversion disorder: the modern hysteria. Advances in Psychiatric Treatment 12(2): 152-157.
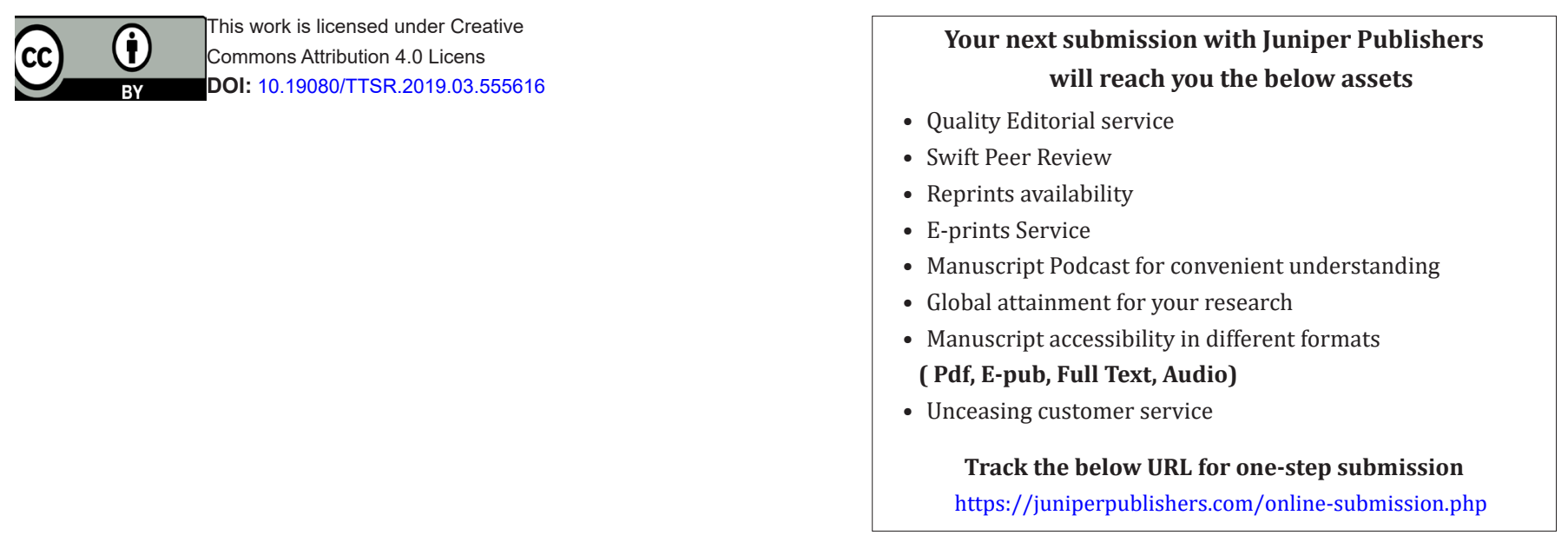